\title{
Estimates for operators on weighted Morrey spaces and their applications to nondivergence elliptic equations
}

\author{
Shaoguang Shi ${ }^{1}$, Zunwei Fu' ${ }^{*}$ and Fayou Zhao ${ }^{2}$
}

\section{"Correspondence:}

zwfu@mail.bnu.edu.cn

'Department of Mathematics, Linyi University, Linyi, 276005, P.R. China

Full list of author information is

available at the end of the article

\begin{abstract}
In this paper, we study the norm inequalities for sublinear operators and their commutators on weighted Morrey spaces. As application, the regularity in the weighted Morrey spaces of strong solutions to nondivergence elliptic equations with VMO coefficients is considered.

MSC: 42B20; 35J25

Keywords: weighted Morrey space; sublinear operator; nondivergence elliptic equation; commutator
\end{abstract}

\section{Introduction and main results}

It is well known that Morrey first introduced the classical Morrey spaces to investigate the local behavior of solutions to second-order elliptic partial differential equations (PDEs) in [1]. In recent years there has been an explosion of interest in the study of the boundedness of operators on Morrey-type spaces. It was found that many properties of solutions to PDEs are concerned with the boundedness of some operators on Morrey-type spaces. In fact, the better inclusion between Morrey and Hölder spaces permits to obtain higher regularity of the solutions to different elliptic and parabolic boundary problems. Given $f \in L_{l o c}^{p}\left(\mathbb{R}^{n}\right)$ and $1 \leq p \leq q<\infty$, Morrey spaces are defined by (cf. [2])

$$
M_{p, q}\left(\mathbb{R}^{n}\right)=\left\{f:\|f\|_{M_{p, q}\left(\mathbb{R}^{n}\right)}=\sup _{B}\left(\frac{1}{|B|^{1-\frac{p}{q}}} \int_{B}|f(x)|^{p} d x\right)^{\frac{1}{p}}<\infty\right\},
$$

where the supremum is taken over all the balls in $\mathbb{R}^{n}$. Obviously, $M_{p, p}\left(\mathbb{R}^{n}\right)=L^{p}\left(\mathbb{R}^{n}\right)$. For more connections between Morrey spaces and some other function spaces, see [3].

We will introduce two important operators including the Hardy-Littlewood maximal operator and the Calderón-Zygmund singular integral operator. Given $f \in L_{l o c}\left(\mathbb{R}^{n}\right)$, the Hardy-Littlewood maximal operator $M$ is defined by

$$
M f(x)=\sup _{B \ni x} \frac{1}{|B|} \int_{B}|f(y)| d y, \quad x \in \mathbb{R}^{n} .
$$

The Calderón-Zygmund singular integral operator is defined by

$$
T f(x)=\text { p.v. } \int_{\mathbb{R}^{n}} K(x-y) f(y) d y,
$$

@ 2013 Shi et al.; licensee Springer. This is an Open Access article distributed under the terms of the Creative Commons Attribution License (http://creativecommons.org/licenses/by/2.0), which permits unrestricted use, distribution, and reproduction in any medium, provided the original work is properly cited. 
where $K$ is the general Calderón-Zygmund kernel satisfying the following conditions:

$$
|K(x)| \leq \frac{C}{|x|^{n}}, \quad|\nabla K(x)| \leq \frac{C}{|x|^{n+1}}, \quad x \neq 0
$$

and

$$
\int_{r \leq|x| \leq R} K(x) d x=0, \quad 0<r<R<\infty .
$$

The operators $M$ and $T$ play a key role in harmonic analysis since the operator $M$ could control much crucial quantitative information concerning the given functions despite their larger size, while the operator $T$, with Hilbert transform as its prototype, is closely connected with PDEs, operator theory and other fields; see [4] for more details. In 1987, Chiarenza and Frasca [2] obtained the boundedness of $M$ and $T$ on $M_{p, q}\left(\mathbb{R}^{n}\right)$. For the boundedness of operators in Morrey spaces on homogeneous spaces, see [5]. For some results on the boundedness for the multilinear singular integral operators on Morrey-type spaces, see [6].

Weighted inequalities arise naturally in Fourier analysis, but their use is best justified by the variety of applications in which they appear. For example, the theory of weights is of great importance in the study of boundary value problems for Laplace's equations on Lipschitz domains. Other applications of weighted inequalities include extrapolation theory, vector-valued inequalities, and estimates for certain classes of nonlinear mathematical physics equations (see [4]). It is worth pointing out that many authors are interested in the weighted norm inequalities when the weight function belongs to the Muckenhoupt classes. Let $w(x) \geq 0$ and $w(x) \in L_{\text {loc }}\left(\mathbb{R}^{n}\right)$. We say that $w \in A_{p}$ (the Muckenhoupt class) for $1<p<\infty$ if there is a constant $C>0$ such that

$$
\sup _{B \subset \mathbb{R}^{n}}\left(\frac{1}{|B|} \int_{B} w(x) d x\right)\left(\frac{1}{|B|} \int_{B} w(x)^{1-p^{\prime}} d x\right)^{p-1}<\infty,
$$

where $1 / p+1 / p^{\prime}=1 . w \in A_{1}$ if there is a constant $C>0$ such that $M w(x) \leq C w(x)$.

For any nonnegative locally integrable function $w$ and any Lebesgue measurable function $f$, the norm of the weighted Lebesgue space was defined by the norm

$$
\|f\|_{L^{p}(w)}=\left(\int_{\mathbb{R}^{n}}|f(x)|^{p} w(x) d x\right)^{1 / p}, \quad 1 \leq p<\infty .
$$

If $w \equiv 1$, we denote $\|f\|_{L^{p}(w)}$ simply by $\|f\|_{L^{p}\left(\mathbb{R}^{n}\right)}$. It is well known that $M$ and $T$ are bounded operators on $L^{p}(w)$ with $w \in A_{p}(1<p<\infty)$. For the boundedness of sublinear operators on $L^{p}(w)$, see [7].

Komori and Shirai [8] introduced a version of the weighted Morrey space $M_{p, \lambda}(w)$, which is a natural generalization of the weighted Lebesgue space $L^{p}(w)$. Let $1 \leq p<\infty, 0<\lambda<1$ and $w$ be a weight function. Then the spaces $M_{p, \lambda}(w)$ are defined by

$$
M_{p, \lambda}(w)=\left\{f:\|f\|_{M_{p, \lambda}(w)}=\sup _{B \subset \mathbb{R}^{n}}\left(\frac{1}{w(B)^{\lambda}} \int_{B}|f(x)|^{p} w(x) d x\right)^{1 / p}<\infty\right\},
$$

where $w(B)=\int_{B} w(x) d x$. It is obvious that if $w \equiv 1$, then $M_{p, 1-p / q}(w)=M_{p, q}\left(\mathbb{R}^{n}\right)$. For $w \in A_{p}$ $(1 \leq p<\infty), M_{p, 0}(w)=L^{p}(w)$ and $M_{p, 1}(w)=L^{\infty}(w)$. In [8], the authors investigated the 
boundedness of the operators $M$ and $T$ on $M_{p, \lambda}(w)$ with $w \in A_{p}$. Wang and Liu [9] studied the boundedness of the Bochner-Riesz means on $M_{p, \lambda}(w)$ with $w \in A_{p}(1 \leq p<\infty)$. In [10], we discussed the norm inequalities for oscillatory singular integral operators on this space. It is of interest to know whether there is a criterion for the boundedness of operators on $M_{p, \lambda}(w)$, which is the motivation of this paper. The goal of this paper is to extend some known results in [8-10] and to establish the boundedness of some sublinear operators and their commutators on the weighted Morrey spaces under some size conditions. These conditions were first proposed by Li and Yang [11] and are satisfied by most of the operators in harmonic analysis. As applications, the strong solutions of nondivergence elliptic equations with $V M O$ coefficients will be given.

Let $D_{k}=\left\{x \in \mathbb{R}^{n}:|x| \leq 2^{k}\right\}, A_{k}=D_{k} \backslash D_{k-1}$ for $k \in Z$ and $\chi_{E}$ be the characteristic function of the set $E$. Then we can formulate our main theorems as follows.

Theorem 1.1 Suppose that a sublinear operator $\mathcal{T}$ satisfies the size conditions

$$
|\mathcal{T} f(x)| \leq C|x|^{-n} \mid f \|_{L^{1}\left(\mathbb{R}^{n}\right)}
$$

when $\operatorname{supp} f \subseteq A_{k},|x| \geq 2^{k+1}$ with $k \in Z$ and

$$
|\mathcal{T} f(x)| \leq C 2^{-k n}\|f\|_{L^{1}\left(\mathbb{R}^{n}\right)},
$$

when $\operatorname{supp} f \subseteq A_{k},|x| \leq 2^{k-1}$ with $k \in Z$. Let $0<\lambda<1$. Then we have:

(a) If $\mathcal{T}$ is bounded on $L^{p}(w)$ with $w \in A_{p}$, then $\mathcal{T}$ is bounded on $M_{p, \lambda}(w)$, where $1<p<\infty$.

(b) If $\mathcal{T}$ is bounded from $L^{1}(w)$ to $L^{1, \infty}(w)$ with $w \in A_{1}$, then there exists a constant $C>0$ such that for all $\mu>0$ and all $B$,

$$
w(\{x \in B: \mathcal{T} f(x)>\mu\}) \leq C \mu^{-1}\|f\|_{M_{1, \lambda}(w)} w(B)^{\lambda} .
$$

It is easy to check that both $M$ and $T$ satisfy the hypotheses of Theorem 1.1 (see [11, p.488]). Therefore, when $\mathcal{T}$ is $M$ or $T$, Theorem 1.1 agrees with [8, Theorem 3.2] and [8, Theorem 3.2], respectively. If $\mathcal{T}$ is the Bochner-Riesz means, Theorem 1.1 is [9, Theorem 1 , Theorem 2].

Corollary 1.2 Let $1<p<\infty, 0<\lambda<1$ and $w \in A_{p}$. Suppose that a sublinear operator $\overline{\mathcal{T}}$ satisfies the condition

$$
|\overline{\mathcal{T}} f(x)| \leq C \int_{\mathbb{R}^{n}} \frac{|f(y)|}{|x-y|^{n}} d y, \quad x \notin \operatorname{supp} f
$$

for any integrable function $f$ with compact support. If $\overline{\mathcal{T}}$ is bounded on $L^{p}(w)$, then $\overline{\mathcal{T}}$ is bounded on $M_{p, \lambda}(w)$.

If $w \equiv 1$, Corollary 1.2 is [12, Theorem 2.1]. Theorem 1.1 is one of the main results of this paper. It is easy to see that condition (1.3) implies size conditions (1.1) and (1.2) since $|x-y|>|x| / 2$ when $|x| \geq 2^{k+1}$, supp $f \subseteq A_{k}$ while supp $f \subseteq A_{k},|x| \leq 2^{k-1}$ imply that $|x-y|>$ $|y| / 2$. So, the proof of Corollary 1.2 is straightforward; see also Theorem 3 in [13]. 
Condition (1.3) was first introduced by Soria and Weiss [14]. It is worth pointing out that (1.3) is satisfied by many operators in harmonic analysis, such as the Calderón-Zygmund singular integral operator $T$, the Carleson maximal operator, Fefferman's singular multiplier operator, Fefferman's singular integral operator, oscillatory integral of Ricci and Stein [15], Bochner-Riesz means at the critical index, singular integral operators with oscillating kernels and so on. For more details, see [16, Remark 5] and [12, p.427]. Bandaliev [16] studied the boundedness of a certain sublinear operator which satisfies (1.3) on weighted variable Lebesgue spaces. For the boundedness of a certain sublinear operator which satisfies (1.3) on product Hardy spaces, see [17].

The bounded mean oscillation function space $B M O$ was first introduced by John and Nirenberg [18] in the study of regular solutions of elliptic PDEs. A locally integrable function $f$ will be said to belong to $B M O$ if

$$
\|f\|_{B M O}=\sup _{B \ni x} \frac{1}{|B|} \int_{B}\left|f(y)-f_{B}\right| d y<\infty
$$

where $f_{B}=\frac{1}{|B|} \int_{B} f(y) d y$.

The commutator formed by an operator $N$ and a symbol function $b$ is usually defined by $N_{b} f$. The boundedness of $N_{b}$ is worse than $N$ (for example, the singularity, see also [19]). Coifman, Rochberg and Weiss [20] first studied the boundedness of $N_{b}$ in their study of certain factorization theorems for generalized Hardy spaces. They showed that $N_{b} f$ is bounded on $L^{p}\left(\mathbb{R}^{n}\right), 1<p<\infty$, if and only if $b \in B M O$ when $N=T$. Since then many works concerning the topic of commutators of different operators with $B M O$ functions have come into existence. For some of other works, see [21] and [22]. In [8], the authors proved the weighted boundedness for $M_{b}$ and $T_{b}$ on $M_{p, \lambda}(w)$ with $b \in B M O\left(\mathbb{R}^{n}\right)$. We shall extend the corresponding results of the sublinear operator to their commutators. For a sublinear operator $\overline{\mathcal{T}}$, we will make the following assumption on its commutator $\overline{\mathcal{T}}_{b}$ :

$$
\left|\overline{\mathcal{T}}_{b} f(x)\right| \leq C \int_{\mathbb{R}^{n}} \frac{|b(x)-b(y)||f(y)|}{|x-y|^{n}} d y, \quad x \notin \operatorname{supp} f .
$$

Theorem 1.3 Let $1<p<\infty, 0<\lambda<1, w \in A_{p}$, and let a sublinear operator $\overline{\mathcal{T}}$ satisfy (1.3). If $\overline{\mathcal{T}}_{b}$ is bounded on $L^{p}(w)$ with $b \in B M O\left(\mathbb{R}^{n}\right)$, then $\overline{\mathcal{T}}_{b}$ is bounded on $M_{p, \lambda}(w)$.

When $\overline{\mathcal{T}}=T$, Theorem 1.3 agrees with [12, Theorem 2.2] and [8, Theorem 3.4].

Let $0<\alpha<n$. Then the fractional maximal operator and the fractional integral are defined by

$$
M_{\alpha} f(x)=\sup _{B \ni x} \frac{1}{|B|^{1-\alpha / n}} \int_{B}|f(y)| d y, \quad x \in \mathbb{R}^{n}
$$

and

$$
I_{\alpha} f(x)=\int_{\mathbb{R}^{n}} \frac{f(y)}{|x-y|^{n-\alpha}} d y
$$

respectively. An early impetus to the study of fractional integrals originated from the problem of fractional derivation (see [23]). Besides their contributions to harmonic analysis, 
the fractional integrals also play an essential role in many fields. The Hardy-LittlewoodSobolev inequality about the fractional integral is still an indispensable tool to establish time-space estimates for the heat semigroup of nonlinear evolution equations; see [24]. For the fractional case, weighted Morrey spaces with two weights, which are also introduced by Komori and Shirai in [8], will be needed. Suppose that $w(x)$ is a nonnegative locally integrable function on $\mathbb{R}^{n}$. We say that $w \in A_{(p, q)}(1<p, q<\infty)$ if there exists a constant $C>0$ such that

$$
\sup _{B \subset \mathbb{R}^{n}}\left(\frac{1}{|B|} \int_{B} w(x)^{q} d x\right)^{1 / q}\left(\frac{1}{|B|} \int_{B} w(x)^{-p^{\prime}} d x\right)^{1 / p^{\prime}} \leq C
$$

and $w \in A_{(1, q)}(1<q<\infty)$ if there exists a constant $C>0$ such that

$$
\sup _{B \subset \mathbb{R}^{n}}\left(\frac{1}{|B|} \int_{B} w(x)^{q} d x\right)^{1 / q}\left(\operatorname{ess}_{B} \sup _{B} \frac{1}{w(x)}\right) \leq C
$$

The boundedness of $I_{\alpha}$ on $M_{p, q}\left(\mathbb{R}^{n}\right)$ was first established by Adams in [25]. In [26], the authors obtained the corresponding boundedness on weighted Lebesgue spaces for $I_{\alpha}$ with $w \in A_{(p, q)}(1 \leq p, q<\infty)$. Let $1 \leq p<\infty, 0<\lambda<1$. For two weights $w_{1}$ and $w_{2}$, the weighted Morrey spaces with two weights are defined by

$$
M_{p, \lambda}\left(w_{1}, w_{2}\right)=\left\{f:\|f\|_{M_{p, k}\left(w_{1}, w_{2}\right)}=\sup _{B}\left(\frac{1}{w_{2}(B)^{\lambda}} \int_{B}|f(x)|^{p} w_{1}(x) d x\right)^{\frac{1}{p}}<\infty\right\} .
$$

If $w_{1}=w_{2}=w$, then we define $M_{p, \lambda}(w, w)=M_{p, \lambda}(w)$. For the corresponding boundedness of $M_{\alpha}$ and $I_{\alpha}$ on $M_{p, \lambda}\left(w_{1}, w_{2}\right)$, see also [8].

We can get similar results for fractional integrals following the line of Theorem 1.1Theorem 1.3.

Theorem 1.4 Let $0<\alpha<n$ and $0<\lambda<1$. Suppose that a sublinear operator $\mathcal{T}_{\alpha}$ satisfies the size conditions

$$
\left|\mathcal{T}_{\alpha} f(x)\right| \leq C|x|^{-(n-\alpha)}\|f\|_{L^{1}\left(\mathbb{R}^{n}\right)}
$$

when $\operatorname{supp} f \subseteq A_{k},|x| \geq 2^{k+1}$ with $k \in Z$ and

$$
\left|\mathcal{T}_{\alpha} f(x)\right| \leq C 2^{-k(n-\alpha)}\|f\|_{L^{1}\left(\mathbb{R}^{n}\right)}
$$

when $\operatorname{supp} f \subseteq A_{k},|x| \leq 2^{k-1}$ with $k \in Z$. Then we have:

(a) If $\mathcal{T}_{\alpha}$ maps $L^{p}\left(w^{p}\right)$ into $L^{q}\left(w^{q}\right)$ with $w \in A_{(p, q)}$, then $\mathcal{T}_{\alpha}$ is bounded from $M_{p, \lambda}\left(w^{p}, w^{q}\right)$ to $M_{q, q \lambda / p}\left(w^{q}\right)$, where $1<p<n / \alpha, 1 / q=1 / p-\alpha / n$ and $1<p<q<\infty$.

(b) If $\mathcal{T}_{\alpha}$ is bounded from $L^{1}(w)$ to $L^{q, \infty}\left(w^{q}\right)$ with $w \in A_{(1, q)}$, then there exists a constant $C>0$ such that for all $\mu>0$ and any ball $B \subset \mathbb{R}^{n}$,

$$
w\left(\left\{x \in B: \mathcal{T}_{\alpha} f(x)>\mu\right\}\right)^{1 / q} \leq C \mu^{-1}\|f\|_{M_{1, \lambda}(w, w q)} w(B)^{\lambda}
$$

where $1<q<\infty$. 
The fractional maximal operator $M_{\alpha}$ satisfies the hypotheses of Theorem 1.4 since the pointwise inequality $M_{\alpha} f(x) \leq I_{\alpha}(|f|)(x)$ holds for $0<\alpha<n$ (see [11, Remark 2.1]). If we take $\mathcal{T}_{\alpha}=M_{\alpha}$ and $\mathcal{T}_{\alpha}=I_{\alpha}$, then Theorem 1.4 agrees with [8, Theorem 3.5] and [8, Theorem 3.6], respectively.

Theorem 1.5 Let $p, q, \alpha, w, \lambda$ be as in Theorem 1.4, and let a sublinear operator $\overline{\mathcal{T}}_{\alpha}$ satisfy

$$
\left|\overline{\mathcal{T}}_{\alpha} f(x)\right| \leq C \int_{\mathbb{R}^{n}} \frac{|f(y)|}{|x-y|^{n-\alpha}} d y, \quad x \notin \operatorname{supp} f
$$

for any integral function $f$ with compact support. If $\overline{\mathcal{T}}_{\alpha, b}$ maps $L^{p}\left(w^{p}\right)$ into $L^{q}\left(w^{q}\right)$ with $b \in B M O\left(\mathbb{R}^{n}\right)$, then $\overline{\mathcal{T}}_{\alpha, b}$ is bounded from $M_{p, \lambda}\left(w^{p}, w^{q}\right)$ to $M_{q, q \lambda / p}\left(w^{q}\right)$.

We remark that both the fractional integral $I_{\alpha}$ and oscillatory fractional integral of Ricci and Stein [15] are examples of operators which satisfy (1.4). For the corresponding boundedness in unweighted cases of the sublinear operators satisfying (1.4) on Herz spaces, we refer the reader to [27] and [11]. Theorem 1.5 reduces to Theorem 3.7 in [8] when $\overline{\mathcal{T}}_{\alpha}=I_{\alpha}$.

Remark 1.1 As another extension of Hilbert transform, a variety of operators related to the singular integrals for Calderón-Zygmund with homogeneous kernels, but lacking the smoothness required in the classical theory, have been studied. In this case, the kernel of the operator has no regularity, and so the operator is called rough integral operator. For some classical survey works about operators with homogeneous kernels, see [4] and [28] for example. Lu, Yang and Zhou studied certain sublinear operators mentioned above with rough kernels on the generalized Morrey space in [29]. In [30], Shi and Fu obtained the boundedness of these sublinear operators with rough kernels on weighted Morrey spaces.

We end this section with the outline of this paper. Section 2 contains the proofs of Theorem 1.1, Theorem 1.3, Theorem 1.4 and Theorem 1.5. In Section 3, by means of the theories of sublinear operators and their commutators obtained in Section 2, we establish the regularity in weighted Morrey spaces of strong solutions to nondivergence elliptic equations with $V M O$ coefficients.

\section{Proofs of the main results}

As in [30], our methods are adopted from [12] in the case of the Lebesgue measure and from [8] dealing with the classical operators. Before the proof of Theorem 1.1, we give some properties of $A_{p}$ weights, which were also stated in Chapter 9 of [4]. For $\lambda>1$, let $\lambda B$ denote the ball with the same center as $B$ and radius $\lambda$ times the radius of $B$.

Lemma 2.1 Let $1 \leq p<\infty$ and $w \in A_{p}$. Then the following statements are true.

(a) There exists a constant $C$ such that

$$
w(2 B) \leq C w(B) .
$$

(b) There exists a constant $C>1$ such that

$$
w(2 B) \geq C w(B) .
$$


(c) There exist two constants $C$ and $r>1$ such that the following reverse Hölder inequality holds for every ball $B \subset \mathbb{R}^{n}$ :

$$
\left(\frac{1}{|B|} \int_{B} w(x)^{r} d x\right)^{1 / r} \leq C\left(\frac{1}{|B|} \int_{B} w(x) d x\right)
$$

(d) For all $\lambda>1$, we have

$$
w(\lambda B) \leq C \lambda^{n p} w(B)
$$

(e) There exist two constants $C$ and $\delta>0$ such that for any measurable set $Q \subset B$,

$$
\frac{w(Q)}{w(B)} \leq C\left(\frac{|Q|}{|B|}\right)^{\delta}
$$

Proof of Theorem 1.1 Let $1<p<\infty, w \in A_{p}$ and $0<\lambda<1$. We first give the proof of (a), for which it suffices to show that

$$
\frac{1}{w(B)^{\lambda}} \int_{B}|\mathcal{T} f(x)|^{p} w(x) d x \leq C\|f\|_{M_{p, \lambda}(w)}^{p}
$$

Without loss of generality, we can assume $r=1$ for a fixed ball $B=B\left(x_{0}, r\right)$ and decompose $f=f \chi_{2 B}+f \chi_{(2 B)}=: f_{1}+f_{2}$ to get

$$
\begin{aligned}
& \frac{1}{w(B)^{\lambda}} \int_{B}|\mathcal{T} f(x)|^{p} w(x) d x \\
& \quad \leq \frac{C}{w(B)^{\lambda}} \int_{B}\left|\mathcal{T} f_{1}(x)\right|^{p} w(x) d x+\frac{C}{w(B)^{\lambda}} \int_{B}\left|\mathcal{T} f_{2}(x)\right|^{p} w(x) d x \\
& \quad=: I+I I .
\end{aligned}
$$

Using the fact that $\mathcal{T}$ is bounded on $L^{p}(w)$, we have

$$
I \leq \frac{C}{w(B)^{\lambda}} \int_{\mathbb{R}^{n}}\left|\mathcal{T} f_{1}(x)\right|^{p} w(x) d x \leq \frac{C}{w(B)^{\lambda}} \int_{2 B}|f(x)|^{p} w(x) d x \leq C\|f\|_{M_{p, \lambda}(w)}^{p}
$$

We are now in a position to estimate the term $I I$. It follows from $w \in A_{p}$ that

$$
\begin{aligned}
\int_{(2 B)^{c}}|f(y)| d y & \leq C \sum_{k=1}^{\infty} \int_{2^{k+1} B \backslash 2^{k} B}|f(y)| d y \\
& \leq C \sum_{k=1}^{\infty}\left(\int_{2^{k+1} B}|f(y)|^{p} w(y) d y\right)^{1 / p}\left(\int_{2^{k+1} B} w(y)^{-p^{\prime} / p} d y\right)^{1 / p^{\prime}} \\
& \leq C\|f\|_{M_{p, \lambda}(w)} \sum_{k=1}^{\infty} \frac{\left|2^{k+1} B\right|}{w\left(2^{k+1} B\right)^{(1-\lambda) / p}} .
\end{aligned}
$$


By assumption (1.2), we have

$$
\begin{aligned}
I I & \leq \frac{C}{w(B)^{\lambda}} \sum_{k=1}^{\infty} 2^{-k n p} \int_{B}\left\|f_{2}\right\|_{L^{1}\left(D_{k}\right)}^{p} w(x) d x \\
& \leq \frac{C}{w(B)^{\lambda-1}} \sum_{k=1}^{\infty} 2^{-k n p}\left(\int_{D_{k}}|f(y)| d y\right)^{p} \\
& \leq C\|f\|_{M_{p, \lambda}(w)}^{p}\left(\sum_{k=1}^{\infty} \frac{w(B)^{(1-\lambda) / p}}{w\left(2^{k+1} B\right)^{(1-\lambda) / p}}\right)^{p} \leq C\|f\|_{M_{p, \lambda}(w)}^{p}
\end{aligned}
$$

Here we have used (2.2) in the last inequality. Combining (2.6) with (2.7), we get (2.5).

We can now proceed analogously to the proof of part (b). We will show the following inequality:

$$
\sup _{\mu>0} \frac{\mu}{w(B)^{\lambda}} w(\{x \in B:|\mathcal{T} f(x)|>\mu\}) \leq C\|f\|_{M_{1, \lambda}(w)}^{p}
$$

Decompose $f=f \chi_{2 B}+f \chi_{(2 B)^{c}}=: f_{1}+f_{2}$ with $B$ as that of (a) to obtain

$$
\begin{aligned}
& w(\{x \in B:|\mathcal{T} f(x)|>\mu\}) \\
& \quad \leq w\left(\left\{x \in B:\left|\mathcal{T} f_{1}(x)\right|>\mu / 2\right\}\right)+w\left(\left\{x \in B:\left|\mathcal{T} f_{2}(x)\right|>\mu / 2\right\}\right) \\
& \quad=: J+J J .
\end{aligned}
$$

An application of $(2.1)$ and the weighted weak $(1,1)$ type estimates for $\mathcal{T}$ yield that

$$
J \leq w\left(\left\{x \in \mathbb{R}^{n}:\left|\mathcal{T} f_{1}(x)\right|>\mu / 2\right\}\right) \leq C \mu^{-1}\|f\|_{M_{1, \lambda}}(w) w(B)^{\lambda}
$$

For the term $J J$, an elementary estimate shows

$$
J \leq \frac{C}{\mu} \int_{\left\{x \in B:\left|\mathcal{T} f_{2}(x)\right|>\mu / 2\right\}}\left|\mathcal{T} f_{2}(x)\right| w(x) d x .
$$

On the other hand, a further use of (1.2) yields

$$
\left|\mathcal{T} f_{2}(x)\right| \leq C \sum_{k=1}^{\infty} 2^{-k n} \int_{D_{k}}|f(y)| d y \leq C \sum_{k=1}^{\infty} 2^{-k n} \int_{2^{k+1} B}|f(y)| d y,
$$

from which it follows that

$$
\begin{aligned}
J & \leq \frac{C}{\mu} \sum_{k=1}^{\infty} 2^{-k n} \int_{2^{k+1} B}|f(y)| w(y) d y \leq \frac{C}{\mu}\|f\|_{M_{1, \lambda}}(w) \sum_{k=1}^{\infty} 2^{k n(\lambda-1)} w(B)^{\lambda} \\
& \leq \frac{C}{\mu}\|f\|_{M_{1, \lambda}}(w) w(B)^{\lambda} .
\end{aligned}
$$

We have thus completed the proof of (b).

The proof of Theorem 1.3 depends heavily on the following remarks about $B M O$ functions. 
Lemma 2.2 [31, Theorem 3.8] (see also [4, Proposition 7.1.5]) Let $1 \leq p<\infty, b \in$ $B M O\left(\mathbb{R}^{n}\right)$. Then, for any ball $B \subset \mathbb{R}^{n}$, the following statements are true:

(a) There exist constants $C_{1}, C_{2}$ such that for all $\alpha>0$,

$$
\left|\left\{x \in B:\left|b(x)-b_{B}\right|>\alpha\right\}\right| \leq C_{1}|B| e^{-C_{2} \alpha /\|b\|_{B M O\left(\mathbb{R}^{n}\right)} .}
$$

Inequality (2.8) is called John-Nirenberg inequality.

(b)

$$
\left|b_{2^{\lambda} B}-b_{B}\right| \leq 2^{n} \lambda\|b\|_{B M O\left(\mathbb{R}^{n}\right)} .
$$

Lemma 2.3 [4, Proposition 7.1.2] (see also [32, Theorem 5]) Let $w \in A_{\infty}$ and $1<p<\infty$. Then the following statements are equivalent:

(a) $\|b\|_{B M O\left(\mathbb{R}^{n}\right)} \sim \sup _{B}\left(\frac{1}{|B|} \int_{B}\left|b(x)-b_{B}\right|^{p} d x\right)^{\frac{1}{p}}$;

(b) $\|b\|_{B M O\left(\mathbb{R}^{n}\right)} \sim \sup _{B} \inf _{a \in \mathbb{R}} \frac{1}{|B|} \int_{B}|b(x)-a| d x$;

(c) $\|b\|_{B M O(w)}=\sup _{B} \frac{1}{w(B)} \int_{B}\left|b(x)-b_{B, w}\right| w(x) d x$, where $B M O(w)=\left\{b:\|b\|_{B M O(w)}<\infty\right\}$ and $b_{B, w}=\frac{1}{w(B)} \int_{B} b(y) w(y) d y$.

Lemma 2.4 Let $b \in B M O\left(\mathbb{R}^{n}\right), w \in A_{p}, B=B\left(x_{0}, r\right)$ be a fixed ball, $0<\lambda<1$ and $1<p<\infty$. Then the inequality

$$
\left(\int_{\left|x_{0}-y\right|>2 r} \frac{|f(y)|}{\left|x_{0}-y\right|^{n}}\left|b_{B, w}-b(y)\right| d y\right)^{p} w(B)^{1-\lambda} \leq C\|f\|_{M_{p, \lambda}(w)}^{p}\|b\|_{B M O\left(\mathbb{R}^{n}\right)}^{p}
$$

holds for every $y \in(2 B)^{c}$ and $f \in M_{p, \lambda}(w)$, where $(2 B)^{c}=\mathbb{R}^{n} \backslash 2 B$.

Proof The proof of Lemma 2.4 has a root in [30], which we adopted here for the completeness of this paper. Applying Hölder's inequality to the left-hand side of (2.10), we obtain

$$
\begin{aligned}
& \left(\int_{\left|x_{0}-y\right|>2 r} \frac{|f(y)|}{\left|x_{0}-y\right|^{n}}\left|b_{B, w}-b(y)\right| d y\right)^{p} w(B)^{1-\lambda} \\
& \leq\left(\sum_{j=1}^{\infty} \int_{2^{j_{r<}\left|x_{0}-y\right|<j^{j+1} r}} \frac{|f(y)|}{\left|x_{0}-y\right|^{n}}\left|b_{B, w}-b(y)\right| d y\right)^{p} w(B)^{1-\lambda} \\
& \leq\left(\sum_{j=1}^{\infty} \frac{1}{\left|2^{j} B\right|} \int_{2^{j+1} B}|f(y)|\left|b_{B, w}-b(y)\right| d y\right)^{p} w(B)^{1-\lambda} \\
& \leq C\left[\sum_{j=1}^{\infty} \frac{1}{\left|2^{j} B\right|}\left(\int_{2^{j+1} B}|f(y)|^{p} w(y) d y\right)^{1 / p}\left(\int_{2^{j+1} B}\left|b_{B, w}-b(y)\right|^{p^{\prime}} w(y)^{1-p^{\prime}} d y\right)^{1 / p^{\prime}}\right]^{p} \\
& \quad \times w(B)^{1-\lambda} \\
& \leq C\|f\|_{M_{p, \lambda}(w)}^{p}\left[\sum_{j=1}^{\infty} \frac{w\left(2^{j+1} B\right)^{\frac{\lambda}{p}}}{\left|2^{j} B\right|}\left(\int_{2^{j+1} B}\left|b_{B, w}-b(y)\right|^{p^{\prime}} w(y)^{1-p^{\prime}} d y\right)^{1 / p^{\prime}}\right]^{p} w(B)^{1-\lambda} .
\end{aligned}
$$

For the simplicity, we define

$$
\mathcal{I}=\left(\int_{2^{j+1} B}\left|b_{B, w}-b(y)\right|^{p^{\prime}} w(y)^{1-p^{\prime}} d y\right)^{1 / p^{\prime}} .
$$


By an elementary estimate, we have

$$
\begin{aligned}
\mathcal{I} & \leq\left(\int_{2^{j+1} B}\left(\left|b_{2^{j+1} B, w^{1-p^{\prime}}}-b(y)\right|+\left|b_{2^{j+1} B, w^{1-p^{\prime}}}-b_{B, w}\right|\right)^{p^{\prime}} w(y)^{1-p^{\prime}} d y\right)^{1 / p^{\prime}} \\
& \leq\left(\int_{2^{j+1} B}\left|b_{2^{j+1} B, w^{1-p^{\prime}}}-b(y)\right| w(y)^{1-p^{\prime}} d y\right)^{\frac{1}{p^{\prime}}}+\left|b_{2^{j+1} B, w^{1-p^{\prime}}}-b_{B, w}\right| w^{1-p^{\prime}}\left(2^{j+1} B\right)^{1 / p^{\prime}} \\
& =: \mathcal{I}_{1}+\mathcal{I}_{2} .
\end{aligned}
$$

For the term $\mathcal{I}_{1}$, we use the fact that if $w \in A_{p}$, then $w^{1-p^{\prime}} \in A_{p^{\prime}}$. By Lemma 2.3,

$$
\mathcal{I}_{1} \leq C\|b\|_{B M O\left(w^{1-p^{\prime}}\right)} w^{1-p^{\prime}}\left(2^{j+1} B\right)^{1 / p^{\prime}} \leq C w^{1-p^{\prime}}\left(2^{j+1} B\right)^{1 / p^{\prime}}
$$

To deal with $\mathcal{I}_{2}$, by (2.9), we have

$$
\begin{aligned}
& \left|b_{2^{j+1} B, w^{1-p^{\prime}}}-b_{B, w}\right| \\
& \leq\left|b_{2^{j+1} B, w^{1-p^{\prime}}}-b_{2^{j+1} B}\right|+\left|b_{2^{j+1} B}-b_{B}\right|+\left|b_{B}-b_{B, w}\right| \\
& \leq \frac{1}{w^{1-p^{\prime}}\left(2^{j+1} B\right)} \int_{2^{j+1} B}\left|b(y)-b_{2^{j+1} B}\right| w(y)^{1-p^{\prime}} d y+2^{n}(j+1)\|b\|_{B M O\left(\mathbb{R}^{n}\right)} \\
& \quad+\frac{1}{w(B)} \int_{B}\left|b(y)-b_{B}\right| w(y) d y \\
& =: \\
& \mathcal{I}_{21}+\mathcal{I}_{22}+\mathcal{I}_{23} .
\end{aligned}
$$

Using (2.4) and (2.8), we obtain that

$$
\begin{aligned}
\mathcal{I}_{23} & =\frac{1}{w(B)} \int_{0}^{\infty} w\left(\left\{x \in B:\left|b(y)-b_{B}\right|>\alpha\right\}\right) d \alpha \\
& \leq C \int_{0}^{\infty} e^{-C_{2} \alpha \delta /\|b\|_{B M O\left(\mathbb{R}^{n}\right)}} d \alpha \leq C,
\end{aligned}
$$

and analogously, $\mathcal{I}_{21} \leq C$.

Hence

$$
\mathcal{I}_{2} \leq C\left(2^{n}(j+1)+2\right) w^{1-p^{\prime}}\left(2^{j+1} B\right)^{1 / p^{\prime}}
$$

As a by-product of (2.11) and (2.12), we have

$$
\mathcal{I} \leq C(j+1) w^{1-p^{\prime}}\left(2^{j+1} B\right)^{1 / p^{\prime}}
$$

Then, the proof of (2.10) is concluded from (2.2) and the following observation:

$$
\begin{aligned}
& {\left[\sum_{j=1}^{\infty} \frac{w\left(2^{j+1} B\right)^{\lambda / p}}{\left|2^{j} B\right|}\left(\int_{2^{j+1} B}\left|b(y)-b_{B, w}\right|^{p^{\prime}} w(y)^{1-p^{\prime}} d y\right)^{1 / p^{\prime}}\right]^{p} w(B)^{1-\lambda}} \\
& \quad \leq C\left[\sum_{j=1}^{\infty} \frac{(j+1) w(B)^{(1-\lambda) / p}}{w\left(2^{j+1} B\right)^{(1-\lambda) / p}}\right]^{p}=C .
\end{aligned}
$$


Proof of Theorem 1.3 It is sufficient to show that for a fixed ball $B=B\left(x_{0}, 1\right)$,

$$
\frac{1}{w(B)^{\lambda}} \int_{B}\left|\overline{\mathcal{T}}_{b} f(x)\right|^{p} w(x) d x \leq C\|f\|_{M_{p, \lambda}(w)}^{p}
$$

Decompose $f=f \chi_{2 B}+f \chi_{(2 B)^{c}}=: f_{1}+f_{2}$. Then

$$
\begin{aligned}
\int_{B}\left|\overline{\mathcal{T}}_{b} f(x)\right|^{p} w(x) d x & \leq C\left(\int_{B}\left|\overline{\mathcal{T}}_{b} f_{1}(x)\right|^{p} w(x) d x+\int_{B}\left|\overline{\mathcal{T}}_{b} f_{2}(x)\right|^{p} w(x) d x\right) \\
& =: K+K K .
\end{aligned}
$$

The $L^{p}(w)$ boundedness of $\overline{\mathcal{T}}_{b}$ allows us to get

$$
K \leq C \int_{2 B}|f(x)|^{p} w(x) d x \leq C\|f\|_{M_{p, \lambda}(w)}^{p} w(B)^{\lambda} .
$$

Making use of (1.3), we have

$$
\begin{aligned}
\left|\overline{\mathcal{T}}_{b} f_{2}(x)\right|^{p} & \leq C\left(\int_{\mathbb{R}^{n}} \frac{\left|f_{2}(y)\right||b(x)-b(y)|}{|x-y|^{n}} d y\right)^{p} \\
& \leq C\left(\int_{\left|x_{0}-y\right|>2} \frac{|f(y)|}{\left|x_{0}-y\right|^{n}}\left\{\left|b(x)-b_{B, w}\right|+\left|b_{B, w}-b(y)\right|\right\} d y\right)^{p} .
\end{aligned}
$$

Hence,

$$
\begin{aligned}
K K \leq & C\left(\int_{\left|x_{0}-y\right|>2} \frac{|f(y)|}{\left|x_{0}-y\right|^{n}} d y\right)^{p} \int_{B}\left|b(x)-b_{B, w}\right|^{p} w(x) d x \\
& +C\left(\int_{\left|x_{0}-y\right|>2} \frac{|f(y)|}{\left|x_{0}-y\right|^{n}}\left|b(y)-b_{B, w}\right| d y\right)^{p} w(B) \\
=: & K K_{1}+K K_{2} .
\end{aligned}
$$

From Lemma 2.4, we can obtain $K K_{2} \leq C\|f\|_{M_{p, \lambda}(w)}^{p} w(B)^{\lambda}$.

To estimate $K K_{1}$, we take into account (2.1), (2.3) and Lemma 2.3. Indeed,

$$
\begin{aligned}
K K_{1}= & C\left(\sum_{j=1}^{\infty} \int_{2^{j}<\left|x_{0}-y\right|<2^{j+1}} \frac{|f(y)|}{\left|x_{0}-y\right|^{n}} d y\right)^{p} \int_{B}\left|b(x)-b_{B, w}\right|^{p} w(x) d x \\
\leq & C \sum_{j=1}^{\infty} \frac{1}{\left|2^{j} B\right|}\left(\frac{1}{w\left(2^{j+1} B\right)^{\lambda}} \int_{2^{j+1} B}|f(y)|^{p} w(y) d y\right)^{1 / p} \\
& \times w\left(2^{j+1} B\right)^{\lambda / p}\left(\int_{2^{j+1} B} w(y)^{-1 / p-1} d y\right)^{(p-1) / p} \int_{B}\left|b(x)-b_{B, w}\right|^{p} w(x) d x \\
\leq & C\|f\|_{M_{p, \lambda}(w)}\left(\sum_{j=1}^{\infty} \frac{\left|2^{j+1} B\right|^{-\frac{1}{p}}}{\left|2^{j} B\right|}\left(\frac{1}{\left|2^{j+1} B\right|} \int_{2^{j+1} B} w(y) d y\right)^{-1 / p} w\left(2^{j+1} B\right)^{\lambda / p}\right)^{p} \\
& \times \int_{B}\left|b(x)-b_{B, w}\right|^{p} w(x) d x
\end{aligned}
$$




$$
\begin{aligned}
& \leq C\|f\|_{M_{p, \lambda}(w)}^{p}\|b\|_{B M O\left(\mathbb{R}^{n}\right)}^{p} \sum_{j=1}^{\infty}\left(\frac{w(B)^{(1-\lambda) / p}}{w\left(2^{j+1} B\right)^{(1-k) / p}}\right)^{p} w(B)^{\lambda} \\
& \leq C\|f\|_{M_{p, \lambda}(w)}^{p} w(B)^{\lambda} .
\end{aligned}
$$

Therefore,

$$
K K \leq C\|f\|_{M^{p, \lambda(w)}}^{p} w(B)^{\lambda} .
$$

Combining (2.14) with (2.15), we obtain (2.13). Therefore, we finish the proof of Theorem 1.3.

Proof of Theorem 1.4 We can use similar arguments as in the proof of Theorem 1.1. For the proof of (a), it suffices to show that

$$
\frac{1}{w^{q}(B)^{q \lambda / p}} \int_{B}\left|\mathcal{T}_{\alpha} f(x)\right|^{q} w(x)^{q} d x \leq C\|f\|_{M_{p, \lambda}\left(w^{p}, w^{q}\right)}^{q}
$$

For a fixed ball $B=B\left(x_{0}, 1\right)$, we decompose $f=f \chi_{2 B}+f \chi_{(2 B)^{c}}=: f_{1}+f_{2}$. Since $\mathcal{T}_{\alpha}$ is a sublinear operator, we get

$$
\begin{aligned}
& \frac{1}{w^{q}(B)^{q \lambda / p}} \int_{B}\left|\mathcal{T}_{\alpha} f(x)\right|^{q} w(x)^{q} d x \\
& \quad \leq \frac{C}{w^{q}(B)^{q \lambda / p}} \int_{B}\left(\left|\mathcal{T}_{\alpha} f_{1}(x)\right|^{q}+\left|\mathcal{T}_{\alpha} f_{2}(x)\right|^{q}\right) w^{q}(x) d x \\
& \quad=: L+L L .
\end{aligned}
$$

To estimate the term $L$, using the fact that $\mathcal{T}_{\alpha}$ is bounded from $L^{p}\left(w^{p}\right)$ to $L^{q}\left(w^{q}\right)$ with $w \in A_{(p, q)}$, we can get

$$
\int_{B}\left|\mathcal{T}_{\alpha} f_{1}(x)\right|^{q} w^{q}(x) d x \leq C\|f\|_{M_{p, \lambda}\left(w^{p}, w^{q}\right)}^{q} w^{q}(B)^{q \lambda / p},
$$

which implies that $L \leq C\|f\|_{M_{p, \lambda}\left(w^{p}, w^{q}\right)}$.

For the term $L L$, by similar arguments to those of Theorem 1.1, we obtain

$$
\begin{aligned}
L L & \leq C \sum_{k}\left(2^{-k(n-\alpha)} \int_{A_{k}}|f(y)| d y\right)^{q} w^{q}(B)^{1-q \lambda / p} \\
& \leq C \sum_{k}\left(2^{-k(n-\alpha)}\|f\|_{M_{p, \lambda}\left(w^{p}, w^{q}\right)}\left|2^{k+1} B\right|^{1-\alpha / n} \frac{1}{w^{q}\left(2^{k+1} B\right)^{1 / q-\lambda / p}}\right)^{q} w^{q}(B)^{1-q \lambda / p} \\
& \leq C\|f\|_{M_{p, \lambda}\left(w^{p}, w^{q}\right)}^{q}\left(\sum_{k=1}^{\infty} \frac{w^{q}(B)^{(1 / q-\lambda / p)}}{w^{q}\left(2^{k+1} B\right)^{(1 / q-\lambda / p)}}\right)^{q} \\
& \leq C\|f\|_{M_{p, \lambda}\left(w^{p}, w^{q}\right)}^{q} .
\end{aligned}
$$

We have completed the proof of (a).

Using an argument quite similar to the one in the proof of (a), we can prove (b). We omit the proof here. 
Proof of Theorem 1.5 The proof of Theorem 1.5 is similar to that of Theorem 1.3, except using $w \in A_{(p, q)}$.

\section{Applications to nondivergence elliptic equations}

In this section, we shall give some applications of our main results to nondivergence elliptic equations. The Dirichlet problem on the second-order elliptic equation in nondivergence form is

$$
\begin{cases}L u=\sum_{i, j}^{n} a_{i j}(x) u_{x_{i}} u_{x_{j}}=f & \text { a.e. in } \Omega \\ u=0 & \text { on } \partial \Omega\end{cases}
$$

Here $x=\left(x_{1}, \ldots, x_{n}\right) \in \mathbb{R}^{n}, \Omega$ is a bounded domain of $\mathbb{R}^{n}$. The coefficients $\left(a_{i j}\right)_{i, j=1}^{n}$ of $L$ are symmetric and uniformly elliptic, i.e., for some $v \geq 1$ and every $\xi \in \mathbb{R}^{n}, a_{i j}(x)=a_{j i}(x)$ and $v^{-1}|\xi|^{2} \leq \sum_{i, j=1}^{n} a_{i j}(x) \xi_{i} \xi_{j} \leq v|\xi|^{2}$ with a.e. $x \in \Omega$. In [12], Fan, Lu and Yang investigated the regularity in $M_{p, \lambda}(\Omega)$ of the strong solution to (3.1) with $a_{i j} \in V M O(\Omega)$, the space of the functions of vanishing mean oscillation introduced by Sarason in [33]. The main methods of [12] are based on integral representation formulas established in [34] for the second derivatives of the solution $u$ to (3.1), a priori estimate of the solution to (3.1) and on the theories of singular integrals and sublinear commutators in corresponding Morrey spaces.

By extending some theorems of [12] to weighted versions, we can also establish the regularity in weighted Morrey spaces $M_{p, \lambda}$ of strong solutions to problem (3.1).

Theorem 3.1 Let $w \in A_{p}(1<p<\infty), f \in M_{p, \lambda}(w)$ with $0<\lambda<1$. Then (3.1) has a unique solution $u \in W^{2} M_{p, \lambda}(w)$ satisfying

$$
\|u\|_{W^{2} M_{p, \lambda}(w)} \leq C\|f\|_{M_{p, \lambda}(w)}
$$

where $W^{2} M_{p, \lambda}(w)$ is the Sobolev-Morrey space. $u \in W^{2} M_{p, \lambda}(w)$ means $u$ and its distributional derivatives, $u_{x_{i}}, u_{x_{i} x_{j}}(i, j=1, \ldots, n)$ are in $M_{p, \lambda}(w)$.

The proof of Theorem 3.1 is very similar to that of [12], we omit the details. Here, we only take two main results to explain this similarity. All other proofs of the corresponding theorems are straightforward. Firstly, Theorem 1.3 in Section 1 is just the weighted version of important Theorem 2.1 in [12]. Next, we give the proof of another important result (the weighted version of Theorem 2.3 of [12]).

Let $\mathbb{R}_{+}^{n}=\left\{x=\left(x^{\prime}, x_{n}\right): x^{\prime}=\left(x_{1}, \ldots, x_{n-1}\right) \in \mathbb{R}^{n-1}, x_{n}>0\right\}, L_{+}^{p}(w)=L^{p}\left(w, \mathbb{R}_{+}^{n}\right)$ and $M_{p, \lambda}^{+}=$ $M_{p, \lambda}\left(w, \mathbb{R}_{+}^{n}\right)$. To establish the boundary estimates of the solutions to (3.1), we need the following general theorem for sublinear operators.

Theorem 3.2 Let $1<p<\infty, 0<\lambda<1, w \in A_{p}, \tilde{x}=\left(x^{\prime},-x_{n}\right)$ for $x=\left(x^{\prime}, x_{n}\right) \in \mathbb{R}_{+}^{n}$. If a sublinear operator $\mathfrak{T}$ is bounded on $L_{+}^{p}(w)$ for any $f \in L_{+}^{1}(w)$ with compact support and satisfies

$$
|\mathfrak{T} f(x)| \leq C \int_{\mathbb{R}_{+}^{n}} \frac{|f(y)|}{|\tilde{x}-y|^{n}} d y,
$$

then $\mathfrak{T}$ is bounded on $M_{p, \lambda}^{+}(w)$. 
Proof Let $z \in \mathbb{R}_{+}^{n}$ and $\delta>0$. Set $B_{\delta}^{+}(z)=B_{\delta}(z) \cap \mathbb{R}_{+}^{n}$, where $B_{\delta}(z)=\left\{y \in \mathbb{R}^{n}:|z-y|<\delta\right\}$. We consider the following two cases.

Case 1. $0 \leq z_{n}<2 \delta$. In this case, we write

$$
f(y)=f(y) \chi_{B_{2^{4} \delta}^{+}(z)}(y)+\sum_{i=4}^{\infty} f(y) \chi_{B_{2^{i+1}}^{+}(z) / B_{2^{i} \delta}^{+}(z)}(y)=: \sum_{i=3}^{\infty} f_{i}(y) .
$$

Therefore, by the $L_{+}^{p}(w)$ boundedness of $\mathfrak{T}$ and (3.2), we obtain

$$
\begin{aligned}
& \frac{1}{w\left(B_{\delta}^{+}\right)^{\lambda / p}}\left(\int_{B_{\delta}^{+}}|\mathfrak{T} f(x)|^{p} w(x) d x\right)^{1 / p} \\
& \leq \frac{1}{w\left(B_{\delta}^{+}\right)^{\lambda / p}} \sum_{i=3}^{\infty}\left(\int_{B_{\delta}^{+}}\left|\mathfrak{T} f_{i}(x)\right|^{p} w(x) d x\right)^{1 / p} \\
& \leq \frac{C}{w\left(B_{\delta}^{+}\right)^{\lambda / p}}\left\|f_{3}\right\|_{L_{+}^{p}(w)}+\frac{C}{w\left(B_{\delta}^{+}\right)^{\lambda / p}} \sum_{i=4}^{\infty}\left(\int_{B_{\delta}^{+}}\left(\int_{B_{2^{+}+1_{\delta}}(z) / B_{2^{i} \delta}^{+}(z)} \frac{|f(y)|}{|\tilde{x}-y|^{n}} d y\right)^{p} w(x) d x\right)^{1 / p} \\
& \leq C\|f\|_{M_{p, \lambda}^{+}(w)}+C \sum_{i=4}^{\infty} \frac{1}{\left(2^{i} \delta\right)^{n}}\left(\int_{B_{2^{i+1} \delta}^{+}}|f(y)| d y\right) w\left(B_{\delta}^{+}\right)^{(1-\lambda) / p} \\
& \quad \leq C\|f\|_{M_{p, \lambda}^{+}(w)}\left(1+\sum_{i=4}^{\infty} \frac{w\left(B_{\delta}^{+}\right)^{(1-\lambda) / p}}{w\left(B_{2^{i+1} \delta}^{+}\right)^{(1-\lambda) / p}}\right) \\
& \quad \leq C\|f\|_{M_{p, \lambda}^{+}(w) .}
\end{aligned}
$$

In the last inequality, we have used Lemma 2.1.

Case 2. There exists $i \in \mathbb{N}$ such that $2^{i} \delta \leq z_{n}<2^{i+1} \delta$. In this case, we write

$$
f(y)=f(y) \chi_{2_{2^{i+1} \delta}^{+}(z)}(y)+\sum_{j=1}^{\infty} f(y) \chi_{{2^{i+j+4}}_{\delta}^{+}}(z)(y)=: \sum_{j=0}^{\infty} f_{j}(y) .
$$

By (3.2) and Lemma 2.1, we have

$$
\begin{aligned}
& \frac{1}{w\left(B_{\delta}^{+}\right)^{\lambda / p}}\left(\int_{B_{\delta}^{+}}|\mathfrak{T} f(x)|^{p} w(x) d x\right)^{1 / p} \\
& \leq \frac{C}{w\left(B_{\delta}^{+}\right)^{\lambda / p}}\left(\int_{B_{\delta}^{+}}\left(\int_{B_{2^{i+4} \delta}^{+}(z)} \frac{|f(y)|}{|\tilde{x}-y|^{n}} d y\right)^{p} w(x) d x\right)^{1 / p} \\
& \quad+\frac{C}{w\left(B_{\delta}^{+}\right)^{\lambda / p}} \sum_{j=1}^{\infty}\left(\int_{B_{\delta}^{+}}\left(\int_{B_{2^{+}+j+4 \delta}(z) / B_{2^{+}+j+3 \delta}} \frac{|f(z)|}{|\tilde{x}-y|^{n}} d y\right)^{p} w(x) d x\right)^{1 / p} \\
& \leq C\|f\|_{M_{p, \lambda}^{+}(w)}\left(\frac{w\left(B_{\delta}^{+}\right)^{(1-\lambda) / p}}{w\left(B_{2^{i+4} \delta}^{+}\right)^{(1-\lambda) / p}}+\sum_{j=1}^{\infty} \frac{w\left(B_{\delta}^{+}\right)^{(1-\lambda) / p}}{w\left(B_{2^{i+j} \delta}^{+}\right)^{(1-\lambda) / p}}\right) \\
& \leq C\|f\|_{M_{p, \lambda}^{+}(w) .}
\end{aligned}
$$




\section{Authors' contributions}

SS carried out the study for norm inequalities for sublinear operators and drafted the manuscript. ZF carried out the study for norm inequalities for the commutators of certain sublinear operators. FZ participated in the study for the regularity of strong solutions to nondivergence elliptic equations. All authors read and approved the final manuscript.

\section{Author details}

'Department of Mathematics, Linyi University, Linyi, 276005, P.R. China. ${ }^{2}$ Department of Mathematics, Shanghai University, Shanghai, 200444, P.R. China.

\section{Acknowledgements}

The authors thank Professors Shanzhen Lu, Heping Liu and the reviewers for their valuable suggestions. This work was partially supported by NSF of China (Grant Nos. 11271175, 11201287 and 11301249), AMEP of Linyi University and NSF of Shandong Province (Grant No. ZR2012AQ026).

\section{Received: 11 May 2013 Accepted: 1 August 2013 Published: 20 August 2013}

\section{References}

1. Morrey, C: On the solutions of quasi-linear elliptic partial differential equations. Trans. Am. Math. Soc. 43, 126-166 (1938)

2. Chiarenza, F, Frasca, M: Morrey spaces and Hardy-Littlewood maximal function. Rend. Mat. Appl. 7, 273-279 (1987)

3. Yuan, W, Sickel, W, Yang, D: Morrey and Campanato Meet Besov, Lizorkin and Triebel. Lecture Notes in Mathematics, vol. 2005. Springer, Berlin (2010) xii+281 pp

4. Grafakos, L: Classical and Modern Fourier Analysis. Pearson Education, Upper Saddle River (2004)

5. Fan, D, Lu, S, Yang, D: Boundedness of operators in Morrey spaces on homogeneous spaces and its applications. Acta Math. Sin. New Ser. 14, 625-634 (1998)

6. Grafakos, L, Torres, R: Multilinear Calderón-Zygmund theory. Adv. Math. 165, 124-164 (2002)

7. Ding, Y, Yang, D, Zhou, Z: Boundedness of sublinear operators and commutators on $L^{p, w}\left(\mathbb{R}^{n}\right)$. Yokohama Math. J. 46 , 15-27 (1998)

8. Komori, Y, Shirai, S: Weighted Morrey spaces and a singular integral operator. Math. Nachr. 282, 219-231 (2009)

9. Wang, H, Liu, H: Some estimates for Bochner-Risez operators on weighted Morrey spaces. Acta Math. Sci. 55, 551-560 (2012) (in Chinese)

10. Shi, S, Fu, Z, Lu, S: Boundedness of oscillatory integral operators and their commutators on weighted Morrey spaces. Sci. Sin. Math. 43, 147-158 (2013) (in Chinese)

11. Li, X, Yang, D: Boundedness of some sublinear operators on Herz spaces. III. J. Math. 40, 484-501 (1996)

12. Fan, D, Lu, S, Yang, D: Regularity in Morrey spaces of strong solutions to nondivergence elliptic equations with VMO coefficients. Georgian Math. J. 5, 425-440 (1998)

13. Mustafayev, RC: On boundedness of sublinear operators in weighted Morrey spaces. Azerb. J. Math. 2, 66-79 (2012)

14. Soria, F, Weiss, G: A remark on singular integrals and power weights. Indiana Univ. Math. J. 43, 187-204 (1994)

15. Ricci, F, Stein, E: Harmonic analysis on nilpotant groups and singular integrals I: oscillatory integrals. J. Funct. Anal. 73, 179-194 (1987)

16. Bandaliev, R: The boundedness of certain sublinear operator in the weighted variable Lebesgue spaces. Czechoslov. Math. J. 60, 327-337 (2010)

17. Chang, C, Yang, D, Zhou, Y: Boundedness of sublinear operators on product Hardy spaces and its application. J. Math Soc. Jpn. 62, 321-353 (2010)

18. John, F, Nirenberg, L: On functions of bounded mean oscillation. Commun. Pure Appl. Math. 14, 415-426 (1961)

19. Pérez, C: Endpoint estimates for commutators of singular integral operators. J. Funct. Anal. 128, 163-185 (1995)

20. Coifman, R, Rochberg, R, Weiss, G: Factorization theorems for Hardy spaces in several variables. Ann. Math. 103, 611-635 (1976)

21. Lu, G, Lu, S, Yang, D: Singular integrals and commutators on homogeneous groups. Anal. Math. 28, 103-134 (2002)

22. Segovia, C, Tottra, J: Weighted inequalities for commutators of fractional and singular integrals. Publ. Mat. 35, 209-235 (1991)

23. Andersen, K, Sawyer, E: Weighted norm inequalities for the Riemann-Liouville and Weyl fractional integral operators. Trans. Am. Math. Soc. 308, 547-558 (1988)

24. Kato, $T$ : Strong $L^{p}$ solutions of the Navier-Stokes equations in $\mathbb{R}^{m}$ with applications to weak solutions. Math. Z. 187, 471-480 (1984)

25. Adams, D: A note on Riesz potentials. Duke Math. J. 42, 765-778 (1975)

26. Muckenhoupt, B, Wheeden, R: Weighted norm inequalities for fractional integrals. Trans. Am. Math. Soc. 192, 261-274 (1974)

27. Hernandez, E, Yang, D: Interpolation of Herz spaces and applications. Math. Nachr. 205, 69-87 (1999)

28. Lu, S, Ding, Y, Yan, D: Singular Integrals and Related Topics. World Scientific, Singapore (2007)

29. Lu, S, Yang, D, Zhou, Z: Sublinear operators with rough kernel on generalized Morrey spaces. Hokkaido Math. J. 27, 219-232 (1998)

30. Shi, S, Fu, Z: Boundedness of sublinear operators with rough kernels on weighted Morrey spaces. J. Funct. Spaces Appl. 2013, Article ID 784983 (2013)

31. García-Cuerva, J, Rubio de Francia, J: Weighted Norm Inequalities and Related Topics. North-Holland Math. Stud., North-Holland, Amsterdam (1985)

32. Muckenhoupt, B, Wheeden, R: Weighted bounded mean oscillation and the Hilbert transform. Stud. Math. $\mathbf{5 4}$ 221-237 (1976)

33. Sarason, D: On functions of vanishing mean oscillation. Trans. Am. Math. Soc. 207, 391-405 (1975)

34. Chiarenza, F, Frasca, M, Longo, P: W', solvability of the Dirichlet problem for nondivergence elliptic equations with VMO coefficients. Trans. Am. Math. Soc. 336, 841-853 (1993) 
doi:10.1186/1029-242X-2013-390

Cite this article as: Shi et al.: Estimates for operators on weighted Morrey spaces and their applications to nondivergence elliptic equations. Journal of Inequalities and Applications 2013 2013:390.

Submit your manuscript to a SpringerOpen ${ }^{\circ}$ journal and benefit from:

- Convenient online submission

- Rigorous peer review

- Immediate publication on acceptance

- Open access: articles freely available online

- High visibility within the field

- Retaining the copyright to your article

Submit your next manuscript at $\gg$ springeropen.com 